\title{
Elevation of liver function tests after laparoscopic gastrectomy using a Nathanson liver retractor.
}

\section{$\operatorname{AUTHOR}(\mathrm{S})$ :}

Kinjo, Yousuke; Okabe, Hiroshi; Obama, Kazutaka; Tsunoda, Shigeru; Tanaka, Eiji; Sakai, Yoshiharu

\section{CITATION:}

Kinjo, Yousuke ... [et al]. Elevation of liver function tests after laparoscopic gastrectomy using a Nathanson liver retractor.. World journal of surgery 2011, 35(12): 2730-2738

\section{ISSUE DATE:}

2011-12

URL:

http://hdl.handle.net/2433/152372

\section{RIGHT:}

The final publication is available at www.springerlink.com; この論文は 出版社版でありません。引用の際には出版社版をご確認ご利用くださ $\iota_{\circ}$; This is not the published version. Please cite only the published version. 


\section{Article type:}

Original scientific reports

\section{Title:}

Elevation of liver function tests after laparoscopic gastrectomy using a Nathanson liver retractor

Authors:

Yousuke Kinjo, MD, Hiroshi Okabe, MD, PhD, Kazutaka Obama, MD, PhD, Shigeru

Tsunoda, MD, PhD, Eiji Tanaka, MD, PhD, Yoshiharu Sakai, MD, PhD

Affiliation:

Department of Surgery, Graduate School of Medicine, Kyoto University

54 Kawahara-cho, Shogoin, Sakyo-ku, Kyoto, 606-8507, Japan

\section{Corresponding Author:}

Hiroshi OKABE

Department of Surgery, Graduate School of Medicine, Kyoto University

54 Kawahara-cho, Shogoin, Sakyo-ku, Kyoto, 606-8507, Japan

e-mail: hokabe@kuhp.kyoto-u.ac.jp

Phone: +81-75-366-7595, fax: +81-75-366-7642

\section{Sources of funding for research}

None.

\section{Conflicts of interest}

The authors declare no conflicts of interest. 


\begin{abstract}
Background: Although pneumoperitoneum has been suspected as a possible cause of transient elevation of liver function tests (LFTs) after laparoscopic surgery, liver damage by direct retraction could also influence postoperative LFTs. The aim of this study was to clarify whether laparoscopic gastrectomy (LG) using a Nathanson retractor was associated with the postoperative elevation of LFTs compared with open gastrectomy (OG).
\end{abstract}

Methods: A retrospective cohort study of $199 \mathrm{LG}$ and 120 OG patients was conducted. Serum aspartate aminotransferase (AST), alanine aminotransferase (ALT), and total bilirubin were measured before operation and at postoperative days 1,3 , and 7 . The abnormal elevation of LFTs was defined as grade 2 or greater elevation in any parameter. To assess the possible effect of pneumoperitoneum, patients who underwent laparoscopic $(\mathrm{n}=324)$ and open $(\mathrm{n}=56)$ colectomy for colorectal cancer were also compared.

Results: In both LG and OG groups, LFTs were significantly elevated postoperatively compared with baseline values. Mean ALT and total bilirubin levels on days 1, 3, and 7 were significantly higher in the LG than OG group. Abnormal elevation of LFTs was more frequent in the LG than OG group (50 vs. $12 \%$ ). In the multivariate analysis, LG was significantly associated with postoperative liver dysfunction (odds ratio=7.99; 95\% confidence interval 3.69 to 18.85). No significant difference in the LFTs elevation was observed between laparoscopic and open colectomy (6\% and 9\%, respectively).

Conclusions: LG resulted in frequent elevation of LFTs. Care should be taken to minimize the intraoperative liver damage when performing LG using a Nathanson retractor. 
Abbreviations

ALHA: Aberrant left hepatic artery

ALT: Alanine aminotransferase

AST: Aspartate aminotransferase

ASA: American Society of Anesthesiology Classification

CI: Confidence interval

LFTs: Liver function tests

LG: Laparoscopic gastrectomy

LC: Laparoscopic colectomy

NCI-CTCAE: National Cancer Institute Common Terminology Criteria for Adverse Events

OC: Open colectomy

OG: Open gastrectomy

OR: Odds ratio 


\section{Introduction}

To pursue minimal invasive surgery for gastric cancer, laparoscopic gastrectomy (LG) was developed and has been increasingly applied in recent years. Several case series studies reported the safety and clinical benefits of LG, including early postoperative recovery and better cosmetic results [1-3]. A recent phase II feasibility study of LG in Japanese patients has reported low frequency (9.1\%) of surgical overall complications, confirming its safety when performed by experienced surgeons [4].

Although overall surgical morbidity does not seem to increase with LG, some studies have shown transient elevation of liver function tests (LFTs) in a substantial proportion of patients after LG [5-7]. Similar transient alterations in LFTs have been reported in other types of laparoscopic surgery, such as cholecystectomy, colectomy, gastric bypass, and Nissen fundoplication [5, 8-13]. Some studies suggest that the increase in intraabdominal pressure by pneumoperitoneum impairs hepatic venous outlet, and causes transient liver damage [5-7].

Other possible causes of postoperative liver dysfunction in laparoscopic surgery include patient position, anesthetic agents, division of aberrant left hepatic artery (ALHA), and direct liver manipulation [5, 6, 10-12, 14-16]. Among these factors, liver manipulation should have greater impact on LG, because it is critical to retract the overhanging hepatic lateral lobe for lymph node dissection. We have introduced a Nathanson liver retractor in LG to maintain a good operative field. Although some studies of antireflux or bariatric surgery have suggested that this method could cause postoperative liver dysfunction [12], the effect of routine use of the Nathanson liver retractor in LG on postoperative LFTs has not been investigated. 
The aim of this study was to clarify whether LG using a Nathanson retractor was associated with the postoperative elevation of LFTs compared with open gastrectomy (OG) under appropriate adjustment for imbalance of the patient's clinicopathological characteristics and potential confounders. In addition, to evaluate the possible effect of pneumoperitoneum on transient liver dysfunction, the incidence and the extent of liver enzyme elevations between laparoscopic colectomy (LC) and open colectomy (OC) were compared.

\section{Materials and Methods}

\section{Patients}

This study was approved by the ethics committee of Kyoto University. All consecutive 373 patients with histologically proven primary gastric cancer who underwent total or distal gastrectomy in our institution between July 2005 and March 2010 were reviewed. To regulate imbalances in patient's preoperative liver function, the following patients were excluded: (1) those with liver metastasis or primary liver tumors, (2) those with chronic hepatic disease, such as HBs antigen positive, Hepatitis $\mathrm{C}$ virus positive, autoimmune liver disease, and alcoholic hepatitis, and (3) those with grade 2 or greater abnormal increase of aspartate aminotransferase (AST), alanine aminotransferase (ALT), and/or total bilirubin on preoperative laboratory tests as classified according to the National Cancer Institute Common Terminology Criteria for Adverse Events (NCI-CTCAE) version 4.0. As a result, 319 patients were included for the analysis. Among them, 199 patients underwent LG (LG group) and 120 patients underwent OG (OG group). To examine the sole effect of pneumoperitoneum on transient liver dysfunction, all 560 consecutive patients with histologically proven primary colorectal cancer who underwent colectomy in our institution 
during the same period were also reviewed. After exclusion of patients according to the same criteria, the laparoscopic colectomy (LC) and the open colectomy (OC) group comprised 324 and 56 patients, respectively. Patients who required conversion from laparoscopic to open surgery were assigned to the LC group according to the intention to treat principle.

\section{Surgical procedure}

The procedure for LG was previously described in detail [17, 18]. Briefly, the patient was placed in a modified lithotomy position. After the first port was inserted through the umbilicus and $\mathrm{CO}_{2}$ pneumoperitoneum at $8 \mathrm{mmHg}$ was established, four operating ports were placed. A Nathanson liver retractor (Cook Japan, Tokyo, Japan) was inserted and fixed with a retractor holder with an appropriate fashion. During the procedure, the patient was placed in the reverse Trendelenburg's position. Lymph node dissection was carried out with the use of ultrasonically activated coagulating shears (SonoSurg®; Olympus, Tokyo, Japan), and the stomach was resected using endoscopic linear staplers. Intracorporeal reconstruction was then carried out either with the Billroth-I or Roux-en-Y method [14], [19].

OG was performed through an upper transverse laparotomy incision. Lymph node dissection was performed using a monopolar and bipolar electric cautery. A blade type liver retractor for open surgery was routinely used with a retractor holder, when performing dissection of suprapyloric or subcardial lymph nodes. Reconstruction was performed with a Roux-en-Y method in both distal and total gastrectomy.

Radical lymphadenectomy which was defined as D2 or “modified D2" lymph node dissection was performed in both groups. D2 was defined according to the general rules of 
the Japanese Association of Gastric Cancer (13th edition) [20], whereas lymph node stations \#12a and/or \#14v were not dissected in "modified D2". In all cases, prophylactic antibiotics were administered prior to and every three hours during the operation.

\section{Preoperative patient assessment}

All patients had detailed preoperative risk assessments based on clinical presentation, chest radiography, electrocardiography, and pulmonary function tests. Preoperative tumor staging was based on physical examination, radiologic examination (computed tomography and/or magnetic resonance imaging), upper gastrointestinal series, ultrasonography, and endoscopy. Before April 2009, LG was only applied to patients with a tumor depth limited to T3 (SS), and without lymph node metastases. After that period, indication of LG was extended to patients with more advanced diseases.

\section{Data extraction and blood samples}

Data of baseline clinical characteristics, operative parameters, postoperative complications, and pathological reports were extracted from the prospectively maintained database of patients who underwent gastrectomy in our department. Laboratory data were collected from medical charts. Blood samples were obtained before operation and on postoperative days 1, 3, and 7, to measure serum AST, ALT, and total bilirubin levels. Total bilirubin, and AST and ALT activities were measured with an automatic analyzer (TBA200FR, Toshiba, Tokyo, Japan; Dimension, Dade Behring, Germany).

\section{Outcomes}


The main study outcome was the incidence of "abnormal elevation of LFTs". Parameters of liver function (AST, ALT, and total bilirubin) at each time point were classified according to the NCI-CTCAE grading system (version 4.0). "Abnormal elevation" was defined as a grade 2 or greater increase in LFTs at any time point, compared to the baseline. In the CTCAE, a grade 2 increase of AST and ALT was defined as $>3.0-5.0 \times$ upper limit of normal (ULN), and a grade 2 increase of total bilirubin was defined as $>1.5-3.0 \times \mathrm{ULN}$. In our institution, the ULNs of AST and total bilirubin were $33 \mathrm{IU} / \mathrm{L}$ and $1.3 \mathrm{mg} / \mathrm{dL}$, respectively. The ULN of ALT was 42 IU/L for male, and 27 IU/L for female.

Postoperative adverse events, such as pneumonia, pleural effusion, atelectasis, cardiac events (myocardial infarction, heart failure, arrhythmia), anastomotic leakage, anastomotic stenosis, ileus, diarrhea, and wound infection were classified according to the NCI-CTCAE. Postoperative complications were defined as grade 2 or greater adverse events that occurred within 30 days of the operation. The pathological classification of the primary tumor, the degree of lymph node involvement, and the presence of organ metastasis were defined according to the TNM classification (version 7), and R classification was used to describe the extent of residual disease after gastrectomy (R0: no residual tumor, R1: microscopic residual tumor, R2: macroscopic residual tumor) [21]. Thirty-day death was defined as death within 30 days of the operation.

\section{Statistical analysis}

Continuous variables are presented as means $( \pm \mathrm{SD})$, and were compared using Student's t test. Categorical data were compared with the $\chi^{2}$ test. Logistic regression models were used to compare the occurrence of abnormal elevation of LFTs between the LG and OG groups. Univariate and multivariate analyses were performed for independent variables. 
Variables with a $p$ value $<0.20$ in the univariate analysis were included in the multivariate analysis. In the multivariate analysis, a $p$ value $<0.05$ was considered statistically significant. All statistical analyses were performed with JMP version 8.0.2 software (SAS Institute, Cary, NC). 


\section{Results}

\section{Patient characteristics and surgical outcomes}

The clinical and pathologic characteristics of eligible 319 gastric cancer patients are shown in Table 1. There was no significant difference in age, gender, body mass index, alcohol use, and medical history. More patients in the OG group received neoadjuvant chemotherapy (25\% vs $7 \%)$ and underwent total gastrectomy (50\% vs $32 \%)$ than in the LG group. The mean preoperative serum albumin in the LG group was higher than that in the OG group. Reflecting the difference of operative indication, the LG group included fewer patients with an advanced stage, lymph node metastasis, or T3/4 tumors compared with the OG group.

Surgical outcomes are shown in Table 2. LG was performed with significantly less blood loss compared to OG (80 vs $472 \mathrm{~g}, p<0.001$ ), while the operation time for LG was longer than that for OG (301 vs $271 \mathrm{~min}, p=0.049$ ). In addition, hospital stay was significantly shorter in the LG group. There was a higher proportion of LG patients with an earlier stage; R0 was achieved in $96 \%$ of these patients, while $25 \%$ of the OG patients resulted in R1/2 resection. Overall complications were significantly less in the LG group (18 patients; $9 \%)$ than in the OG group (22 patients; $18 \%)(p=0.015)$. Liver infarction was diagnosed by computed tomography in one LG patient (Figure 1)—spiking fever in this patient subsided with antibiotics treatment within a week. One patient in each group underwent reoperation due to anastomotic leakage, and one patient who underwent laparoscopic total gastrectomy had a reoperation due to ischemic perforation of the ileum. The patient was a 77-year-old woman with diabetes mellitus and hypertension, and pathological examination revealed the segmental ischemia of ileum due to thromboembolic occlusion of the mesenteric artery. 
Because we did not manipulate the ileum during LG, the complication was not directly related to the intra-operative procedure.

Details of the clinical and pathological features of 380 colorectal cancer patients are shown in Table 3. There were no significant differences in age, gender, body mass index, tumor site, or operation time between the LC and OC groups. Mean blood loss in the LC group was significantly less than that in the OC group (33 vs $430 \mathrm{~g}, p<0.001$ ). In addition, the LC group included more patients with an earlier pathological tumor stage.

\section{Comparison of abnormal elevation of LFTs between LG and OG}

The laboratory data of LFTs are listed in Table 4 and shown in Figure 2. In both LG and OG groups, AST and ALT levels were significantly elevated after the operation compared with the baseline values and reached peaks on the first postoperative day. AST levels decreased more rapidly than ALT levels in both groups and returned to baseline at day 7 . No increase in either the AST or ALT level was observed in the LC or OC group. Total bilirubin levels were significantly elevated compared to the baseline values in all groups, and gradually decreased to baseline at day 7 .

In comparison between the LG and OG groups, mean AST levels on postoperative days 1 and 3, and mean ALT and total bilirubin levels on days 1, 3, and 7, were significantly higher in the LG group $(p<0.001)$, indicating the delayed recovery of ALT and total bilirubin levels in these patients.

“Abnormal elevation of LFTs", which was defined as a grade 2 or greater elevation in either parameter, was observed in 99 out of the 199 LG patients (50\%). On the other hand, only 14 out of the 120 OG patients (12\%) showed an increased level of LFTs. There was no 
significant difference in the incidence of altered LFTs between LC and OC groups (6\% and 9\%, respectively).

\section{Univariate and multivariate analysis for the abnormal elevation of LFTs}

Univariate and multivariate logistic regression analyses regarding the incidence of abnormal elevation of LFTs were performed in all 319 patients who underwent gastrectomy (Table 5). In the univariate analysis, LG, serum albumin, cholecystectomy, and operation time were significant risk factors related to abnormal LFTs. In the multivariate model including variables with a $p$ value $<0.20$ in the univariate analysis, LG was the only independent risk factor for abnormal elevation of LFTs (adjusted odds ratio $=7.99 ; 95 \%$ confidence interval 3.69 to $18.85 ; p<0.001)$. Age, gender, body mass index, preoperative serum albumin, cholecystectomy, depth of tumor invasion, and lymph node metastasis were not associated with abnormal LFTs.

\section{Postoperative course of patients with elevated LFTs}

Postoperative course of patients with abnormally elevated LFTs after LG (n=99) was compared with that of LG patients without elevated LFTs $(n=100)$. There was no significant difference in postoperative complications ( $10 \%$ vs $8 \%, p=0.605)$. Mean postoperative hospital stay was also similar between the two groups (16 vs 14 days, $p=0.154$ ).

\section{Discussion}

Our study showed that a significantly higher incidence of abnormal elevation of LFTs in the LG group than OG group. Notably, nearly half of the LG patients experienced more than three times elevation of liver enzymes postoperatively. Because this is an 
observational study, careful analysis and interpretation of results is necessary. First, in consideration of confounding factors, we excluded cases of liver tumor or liver disease to reduce the factors affecting postoperative liver function. Second, selection bias between the two groups should be considered. Because LG was applied only for patients with a tumor depth of T3 (SS) in the earlier period of this study, tumor stage or metastatic status were not matched between the LG and OG groups. To control for such imbalance, a logistic regression model was adopted. Even after adjustment for these potential confounders, LG remained the only factor responsible for abnormal elevation of LFTs.

In previous studies, factors such as anesthetic agents, patient position, direct liver manipulation, division of an aberrant left hepatic artery (ALHA), and pneumoperitoneum have been reported as possible causes for transient liver enzyme elevation after laparoscopic surgery $[5,6,10-12,15,16]$. Among these factors, anesthetic agents were the same in both groups in our study. Pneumoperitoneum has been argued to be the main cause of postoperative hepatic dysfunction in several studies regarding laparoscopic surgery [5, 813]. A decreased blood flow in the liver due to the pressure of pneumoperitoneum has been suggested as the possible mechanism. However, a study using transesophageal echocardiography has demonstrated an opposite effect of pneumoperitoneum —an elevated hepatic flow during laparoscopic procedures [22]. Moreover, in a large case series study of more than 1000 laparoscopic cholecystectomies, postoperative LFT was elevated in only $3.9 \%$ of patients [8]. In our study, to exclude the effect of liver manipulation and to investigate the sole effect of pneumoperitoneum on LFTs, patients who underwent LC were examined. Abnormal elevation of LFTs was observed in only 6\% of the LC patients, which is similar to $9 \%$ of the OC patients. A head-up position may cause enhanced reduction of hepatic blood flow under pneumoperitoneum[14]. However, abnormal elevation of LFTs was 
not increased in patients who underwent LC in a head-up position compared to patients in a head-down position in our study (4.4\% vs 5.5\%). These data together strongly suggest that pneumoperitoneum and patient position are not responsible for the postoperative abnormal LFTs in patients who underwent laparoscopic surgery.

Transient changes in LFTs are also observed in patients who undergo OG. Previous studies have indicated that division of the ALHA during lymphadenectomy could affect postoperative LFTs [16, 23]. Although there is controversy whether the ALHA should be preserved during lymphadenectomy, postoperative liver dysfunctions were reported to be transient and resumed within a week. Thus, to ensure en bloc dissection of lymph nodes, we routinely divide the left gastric artery at its root even when the ALHA is recognized during the procedure. Because this policy was equally applied to the LG and OG group, the effect of the division of the ALHA should be the same between the two groups. Nevertheless, only in the LG group, did half of the patients experience abnormal elevation of LFTs postoperatively. Although a frequency of the ALHA ranged from 12 to $20 \%$ in the literature $[3,24,25]$, the ligation of the ALHA alone could not explain the high frequency of liver dysfunction in the LG group.

As Jeong et al. suspected in a recent study, direct liver manipulation is the another possible cause [5-7]. In gastric cancer surgery, liver retraction is essential for performing lymphadenectomy along the lesser curvature, the hepatoduodenal ligament, and the cardiac area. For LG, we introduced a Nathanson liver retractor, which has been applied in laparoscopic upper gastrointestinal surgery in western countries for years. Although this retractor provides a good operative field, elevation of liver enzymes has been reported in some studies of laparoscopic fundoplication or gastric bypass [12, 15]. Among three previous studies examining liver functions after LG, only Shinohara et al. used a Nathanson 
retractor; one study used a snake retractor (Diamond-Flex®; Snowden Pencer, USA) and the other did not mention the liver retraction method. In Shinohara's and our studies using a Nathanson retractor, peak ALT values were 213 and 153, which are much higher than those reported in the other two studies (56 and 89, respectively). These observations suggest that heavier retraction by a Nathanson retractor might cause more severe damage to the liver than the other method. In addition, two studies using a Nathanson retractor required a longer operation time than in the others (333 and 301 vs 270 and $230 \mathrm{~min}$ ). Because operation time was also significantly longer in patients with elevated LFTs in our study, this factor could also adversely affect the postoperative LFTs. In fact in the LG group, abnormal elevation of LFTs was more frequently observed in patients who underwent total gastrectomy which required a longer operation time and heavier retraction compared to patients who underwent distal gastrectomy in our study (60\% vs 45\%).

We experienced a focal liver infarction in the lateral segment in one LG patient. Both injury to the hepatic artery and liver retraction are suspected as causes of liver infarction following gastrectomy [26]. In this patient, ALHA was recognized and divided during surgery, while the proper hepatic artery was safely preserved. Because the infarction was focal and consistent with the area where a Nathanson retractor was placed, ischemia could also be a result of the intense retraction.

In conclusion, the current study demonstrated that LG results in frequent elevation of LFTs compared with OG. Liver retraction by a Nathanson retractor could be a major cause. Although elevated liver enzymes subsided spontaneously and usually do not affect patient recovery, rare complications such as liver infarction could happen. Therefore, care should be taken to minimize the intraoperative liver damage when performing LG, by decreasing the duration of liver retraction or using a gentle retraction method. 


\section{Acknowledgements}

The authors acknowledge the invaluable help with data management by the Division of Clinical Trial Design \& Management, Translational Research Center, Kyoto University Hospital. The authors declare no conflicts of interest. 


\section{References}

1. Yano H, Monden T, Kinuta M, et al (2001) The usefulness of laparoscopy-assisted distal gastrectomy in comparison with that of open distal gastrectomy for early gastric cancer. Gastric Cancer 4:93-97.

2. Shimizu S, Noshiro H, Nagai E, et al (2003) Laparoscopic gastric surgery in a Japanese institution: analysis of the initial 100 procedures. J Am Coll Surg 197:372378.

3. Adachi Y, Suematsu T, Shiraishi N, et al (1999) Quality of life after laparoscopyassisted Billroth I gastrectomy. Ann Surg 229:49-54.

4. Katai H, Sasako M, Fukuda H, et al, Group JGCSS (2010) Safety and feasibility of laparoscopy-assisted distal gastrectomy with suprapancreatic nodal dissection for clinical stage I gastric cancer: a multicenter phase II trial (JCOG 0703). Gastric Cancer 13:238-244.

5. Etoh T, Shiraishi N, Tajima M, et al (2007) Transient liver dysfunction after laparoscopic gastrectomy for gastric cancer patients. World J Surg 31:1115-1120.

6. Jeong GA, Cho GS, Shin EJ, et al (2011) Liver function alterations after laparoscopy-assisted gastrectomy for gastric cancer and its clinical significance. World J Gastroenterol 17:372-378.

7. Shinohara T, Kanaya S, Yoshimura F, et al (2010) A Protective Technique for Retraction of the Liver During Laparoscopic Gastrectomy for Gastric Adenocarcinoma: Using a Penrose Drain. J Gastrointest Surg: In press.

8. Bickel A, Weiar A, Eitan A (2008) Evaluation of liver enzymes following elective laparoscopic cholecystectomy: are they really elevated? J Gastrointest Surg 12:14181421.

9. Guven HE, Oral S (2007) Liver enzyme alterations after laparoscopic cholecystectomy. J Gastrointestin Liver Dis 16:391-394.

10. Kotake Y, Takeda J, Matsumoto M, et al (2001) Subclinical hepatic dysfunction in laparoscopic cholecystectomy and laparoscopic colectomy. Br J Anaesth 87:774-777.

11. Morino M, Giraudo G, Festa V (1998) Alterations in hepatic function during laparoscopic surgery. An experimental clinical study. Surg Endosc 12:968-972.

12. Morris LF, Ragavendra N, Yeh MW (2008) Evidence-based assessment of the role of ultrasonography in the management of benign thyroid nodules. World J Surg 32:1253-1263.

13. Nguyen NT, Braley S, Fleming NW, et al (2003) Comparison of postoperative hepatic function after laparoscopic versus open gastric bypass. Am J Surg 186:40-44.

14. Sato K, Kawamura T, Wakusawa R (2000) Hepatic blood flow and function in elderly patients undergoing laparoscopic cholecystectomy. Anesth Analg 90:1198-1202.

15. Lohlun JC, Guirguis A, Wise L (2004) Elevated liver enzymes following open Rouxen-Y gastric bypass for morbid obesity - does timing of liver retraction affect the rise in the levels of transaminases? Obes Surg 14:505-508.

16. Okano S, Sawai K, Taniguchi H, et al (1993) Aberrant left hepatic artery arising from the left gastric artery and liver function after radical gastrectomy for gastric cancer. World J Surg 17:70-73; discussion 74.

17. Satoh S, Okabe H, Kondo K, et al (2009) Video. A novel laparoscopic approach for safe and simplified suprapancreatic lymph node dissection of gastric cancer. Surg Endosc 23:436-437. 
18. Glantzounis GK, Tsimaris I, Tselepis AD, et al (2005) Alterations in plasma oxidative stress markers after laparoscopic operations of the upper and lower abdomen. Angiology 56:459-465.

19. Okabe H, Obama K, Kan T, et al (2010) Medial approach for laparoscopic total gastrectomy with splenic lymph node dissection. J Am Coll Surg 211:e1-6.

20. Japanese gastric cancer association (1999) Japanese classicication of gastric carcinoma. 13th ed. Kanehara Shuppan, Tokyo, p 1-71.

21. Cancer UIUA (2009) TNM classification of malignant tumors, 7th ed. Wiley, New York.

22. Meierhenrich R, Gauss A, Vandenesch P, et al (2005) The effects of intraabdominally insufflated carbon dioxide on hepatic blood flow during laparoscopic surgery assessed by transesophageal echocardiography. Anesth Analg 100:340-347.

23. Shinohara T, Ohyama S, Muto T, et al (2007) The significance of the aberrant left hepatic artery arising from the left gastric artery at curative gastrectomy for gastric cancer. Eur J Surg Oncol 33:967-971.

24. Lee SW, Shinohara H, Matsuki M, et al (2003) Preoperative simulation of vascular anatomy by three-dimensional computed tomography imaging in laparoscopic gastric cancer surgery. J Am Coll Surg 197:927-936.

25. Covey AM, Brody LA, Maluccio MA, et al (2002) Variant hepatic arterial anatomy revisited: digital subtraction angiography performed in 600 patients. Radiology 224:542-547.

26. Kitagawa T, Iriyama K (1998) Hepatic infarction as a complication of gastric cancer surgery: report of four cases. Surg Today 28:542-546. 


\section{Figure legends}

\section{Fig. 1}

Liver infarction following laparoscopic gastrectomy. Contrast-enhanced computed tomography revealed a wedge-shaped low-attenuation area in the lateral segment on the fourth operative day.

\section{Fig. 2}

Time-course changes in liver function tests following gastrectomy. (A) Aspartate aminotransferase (AST). (B) Alanine aminotransferase (ALT). (C) Total bilirubin. * $p<0.01$ compared with open gastrectomy. 


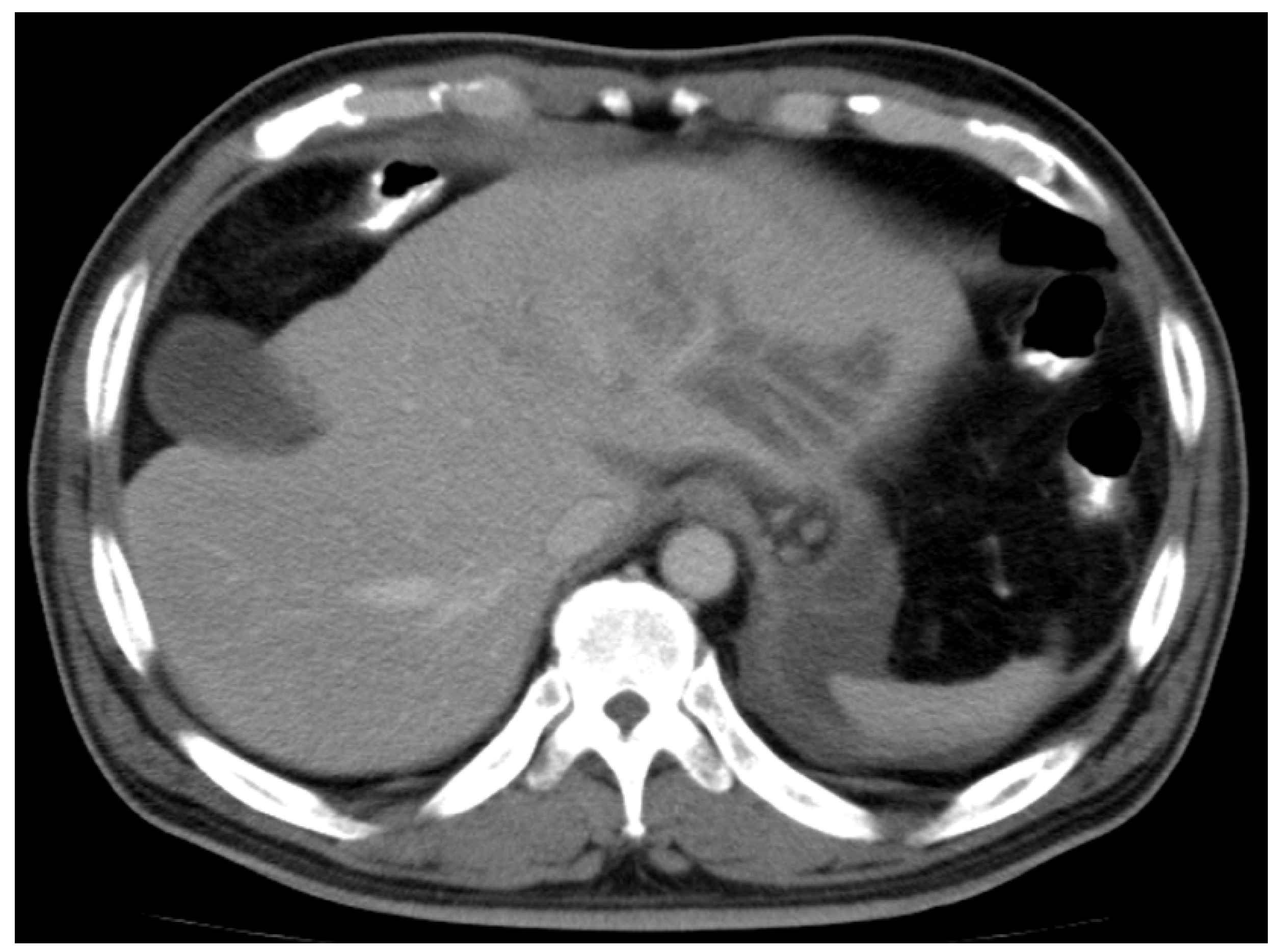




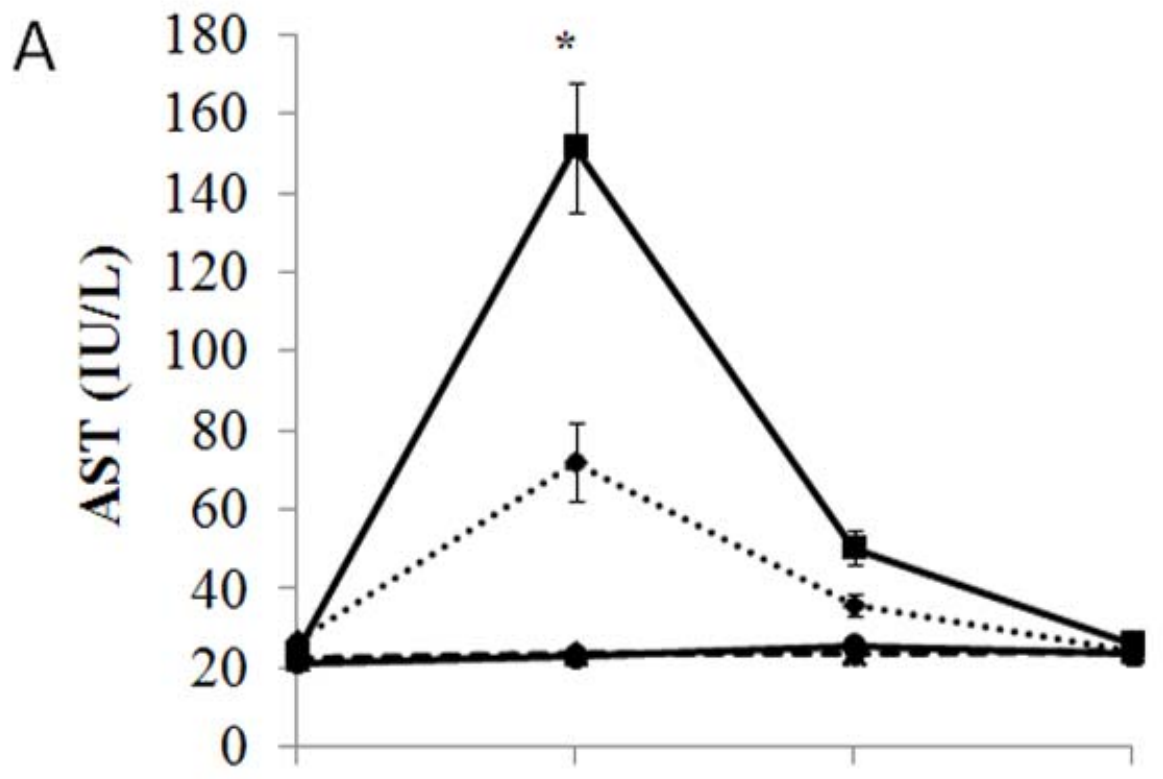

Baseline Day 1 Day3 Day 7

C

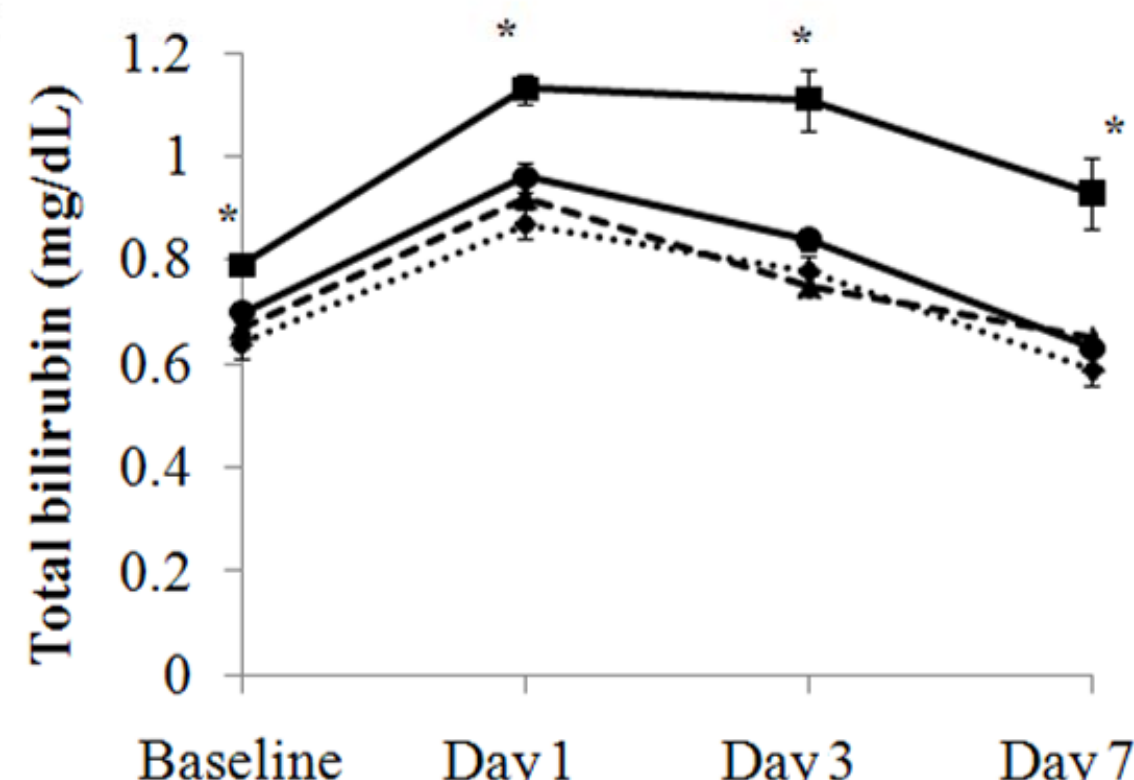

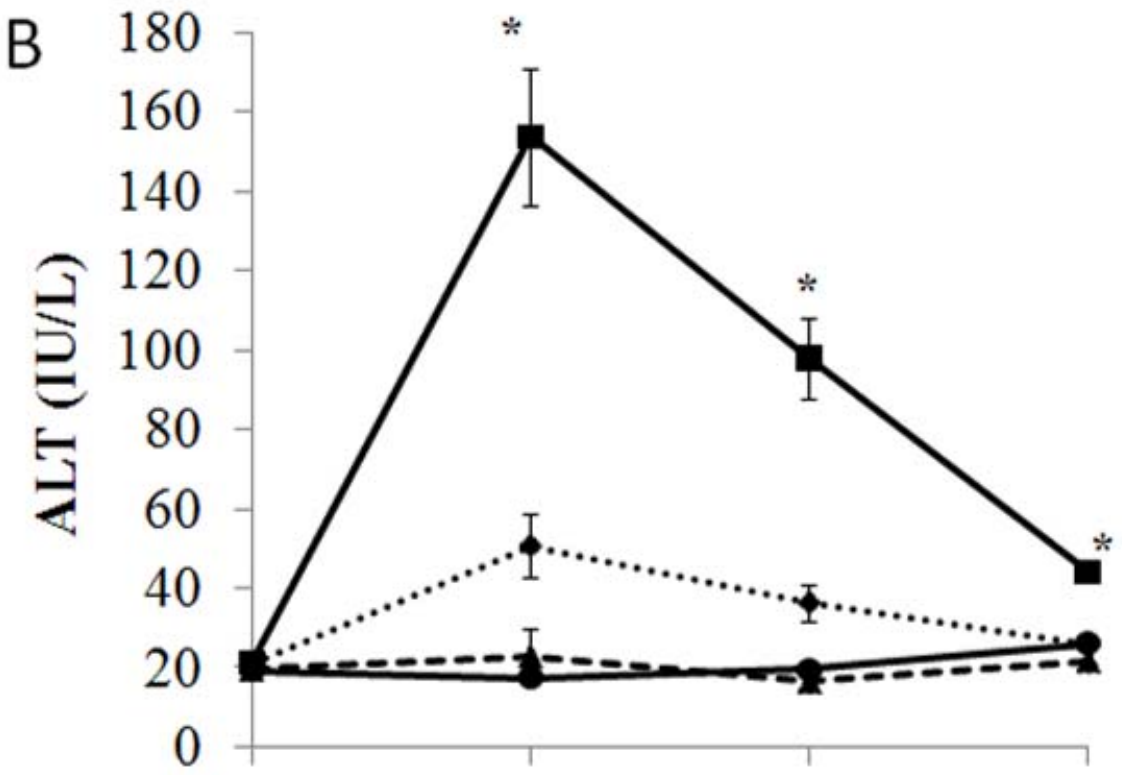

Baseline Day 1 Day 3 Day 7

- Laparoscopic gastrectomy

$\ldots \bowtie$ Open gastrectomy

$\neg$ Laparoscopic colectomy

-๘- Open colectomy 
Table 1 Clinical and pathological characteristics of gastric cancer patients

\begin{tabular}{|c|c|c|c|}
\hline Variable & $\begin{array}{c}\text { LG } \\
(n=199)\end{array}$ & $\begin{array}{c}\text { OG } \\
(n=120)\end{array}$ & $p$ value \\
\hline Age (years)* & $64.0 \pm 11.9$ & $66.5 \pm 11.3$ & 0.073 \\
\hline Gender (male:female) & $125: 74$ & $86: 34$ & 0.106 \\
\hline Body mass index* & $22.0 \pm 3.3$ & $21.5 \pm 3.6$ & 0.203 \\
\hline ASA-PS & & & 0.009 \\
\hline $\mathrm{I}$ & $149(75 \%)$ & $73(61 \%)$ & \\
\hline II & $50(25 \%)$ & $47(39 \%)$ & \\
\hline Alcohol use & $106(53 \%)$ & $65(38 \%)$ & 0.875 \\
\hline Cardiovascular disease & $21(11 \%)$ & $15(13 \%)$ & 0.594 \\
\hline Diabetes mellitus & $34(17 \%)$ & $14(12 \%)$ & 0.190 \\
\hline Serum albumin $(\mathrm{g} / \mathrm{dL}) *$ & $4.1 \pm 0.4$ & $3.8 \pm 0.5$ & $<0.001$ \\
\hline Neoadjuvant chemotherapy & $14(7 \%)$ & $30(25 \%)$ & $<0.001$ \\
\hline Extent of resection & & & 0.001 \\
\hline Total & $63(32 \%)$ & $60(50 \%)$ & \\
\hline Distal & $136(68 \%)$ & $60(50 \%)$ & \\
\hline Cholecystectomy & $10(5 \%)$ & $49(41 \%)$ & $<0.001$ \\
\hline Tumor site & & & 0.128 \\
\hline Upper & $48(24 \%)$ & $39(33 \%)$ & \\
\hline Middle & $78(39 \%)$ & $35(29 \%)$ & \\
\hline Lower & $73(37 \%)$ & $46(38 \%)$ & \\
\hline Tumor stage & & & $<0.001$ \\
\hline $\mathrm{I}$ & $147(73 \%)$ & $31(26 \%)$ & \\
\hline II & $28(14 \%)$ & $22(18 \%)$ & \\
\hline III & $17(9 \%)$ & $31(26 \%)$ & \\
\hline IV & $7(4 \%)$ & $36(30 \%)$ & \\
\hline Depth of tumor invasion & & & $<0.001$ \\
\hline $\mathrm{pT} 1,2$ & $160(80 \%)$ & $41(34 \%)$ & \\
\hline pT3, 4 & $39(20 \%)$ & $79(66 \%)$ & \\
\hline Lymph node metastasis & & & $<0.001$ \\
\hline pN0 & $148(74 \%)$ & $41(34 \%)$ & \\
\hline $\mathrm{pN} 1-3$ & $51(26 \%)$ & $79(66 \%)$ & \\
\hline Extent of node dissection & & & $<0.001$ \\
\hline D1, modified D2 & $108(54 \%)$ & $25(21 \%)$ & \\
\hline D2, D3 & $91(46 \%)$ & $95(79 \%)$ & \\
\hline
\end{tabular}

*Values are means \pm SD. LG: laparoscopic gastrectomy; OG: open gastrectomy; ASA: American Society of Anesthesiology Classification. 
Table 2 Surgical outcomes of gastric cancer patients

\begin{tabular}{lccc}
\hline & LG & OG & \\
& $(\mathrm{n}=199)$ & $(\mathrm{n}=120)$ & $p$ value \\
\hline Operative blood loss $(\mathrm{g}) *$ & $80(5-3600)$ & $472(10-3400)$ & $<0.001$ \\
Operation time (min)* & $301(179-560)$ & $271(60-1480)$ & 0.049 \\
Residual tumor & & & $<0.001$ \\
R0 & $192(96 \%)$ & $90(75 \%)$ & \\
R1-R2 & $7(4 \%)$ & $30(25 \%)$ & \\
Reoperation & $2(1 \%)$ & $1(1 \%)$ & 0.878 \\
Postoperative hospital stay (days)* & $12(7-85)$ & $15(8-71)$ & $<0.001$ \\
Overall surgical complications & $18(9 \%)$ & $22(18 \%)$ & 0.015 \\
Pneumonia & 6 & 4 & \\
Pleural effusion & 2 & 4 & \\
Atelectasis & 4 & 3 & \\
Arrhythmia & 2 & 0 & \\
Enteritis & 0 & 1 & \\
Diarrhea & 0 & 1 & \\
Ileus & 2 & 2 & \\
Anastomotic leakage & 5 & 4 & \\
Anastomotic stenosis & 1 & 0 & \\
Liver infarction & 2 & 2 & \\
Wound infection & 0 & 0 & \\
Death within 30 days & 1 & & \\
\hline
\end{tabular}

*Values are means \pm SD. LG: laparoscopic gastrectomy; OG: open gastrectomy; R0: no residual tumor; R1: microscopic residual tumor; R2: macroscopic residual tumor. 
Table 3 Clinical characteristics and surgical outcomes of colorectal cancer patients

\begin{tabular}{lccc}
\hline & LC & OC & \\
Variable & $(\mathrm{n}=324)$ & $(\mathrm{n}=56)$ & $p$ value \\
\hline Age (years)* & $67.4 \pm 11.0$ & $68.4 \pm 13.9$ & 0.547 \\
Gender (male:female) & $193: 131$ & $33: 23$ & 0.928 \\
Body mass index* & $22.1 \pm 3.3$ & $21.5 \pm 3.3$ & 0.191 \\
Tumor site & & & 0.613 \\
$\quad$ Cecum / Ascending & $56(18 \%)$ & $14(24 \%)$ & \\
Transverse & $27(8 \%)$ & $6(11 \%)$ & \\
Descending & $21(6 \%)$ & $5(9 \%)$ & \\
Sigmoid & $89(27 \%)$ & $10(18 \%)$ & \\
Recto-sigmoid & $35(11 \%)$ & $7(13 \%)$ & \\
Rectum & $96(30 \%)$ & $14(25 \%)$ & \\
Tumor stage & & & \\
0 & $23(7 \%)$ & $5(9 \%)$ & \\
I & $80(25 \%)$ & $4(7 \%)$ & \\
II & $109(34 \%)$ & $26(46 \%)$ & \\
III & $100(30 \%)$ & $11(20 \%)$ & \\
IV & $12(4 \%)$ & $10(18 \%)$ & \\
Operative blood loss $(\mathrm{g}) \dagger$ & $33(0-900)$ & $430(15-7030)$ & $<0.001$ \\
Operation time (min) $\dagger$ & $244(98-637)$ & $234(105-626)$ & \\
\hline Values are meanstSD & & & \\
\hline
\end{tabular}

*Values are means \pm SD. $†$ Values are medians (range). LC: laparoscopic colectomy; OC: open colectomy. 
Table 4 Laboratory data and incidence of abnormal elevation of LFTs

\begin{tabular}{|c|c|c|c|c|c|c|}
\hline & $\begin{array}{c}\mathrm{LG} \\
(\mathrm{n}=199)\end{array}$ & $\begin{array}{c}\mathrm{OG} \\
(\mathrm{n}=120)\end{array}$ & $p 1$ & $\begin{array}{c}\mathrm{LC} \\
(\mathrm{n}=324)\end{array}$ & $\begin{array}{c}\mathrm{OC} \\
(\mathrm{n}=56)\end{array}$ & $p 2$ \\
\hline \multicolumn{7}{|l|}{ AST (IU/dL)* } \\
\hline Baseline & $23.6 \pm 11.1$ & $26.8 \pm 14.2$ & 0.024 & $21.3 \pm 8.5$ & $22.1 \pm 8.5$ & 0.557 \\
\hline Day 1 & $151.7 \pm 231.3$ & $72.1 \pm 109.5$ & $<0.001$ & $23.0 \pm 14.1$ & $23.6 \pm 12.7$ & 0.788 \\
\hline Day 3 & $50.4 \pm 63.2$ & $35.7 \pm 30.4$ & 0.017 & $25.5 \pm 18.4$ & $23.6 \pm 10.3$ & 0.462 \\
\hline Day 7 & $26.2 \pm 13.6$ & $24.5 \pm 14.1$ & 0.287 & $23.7 \pm 15.7$ & $24.2 \pm 23.3$ & 0.842 \\
\hline \multicolumn{7}{|l|}{$\mathrm{ALT}(\mathrm{IU} / \mathrm{dL}) *$} \\
\hline Baseline & $21.4 \pm 13.3$ & $20.9 \pm 12.7$ & 0.751 & $19.3 \pm 12.2$ & $19.5 \pm 12.4$ & 0.904 \\
\hline Day 1 & $153.8 \pm 244.8$ & $50.8 \pm 87.4$ & $<0.001$ & $17.5 \pm 11.9$ & $22.8 \pm 53.8$ & 0.111 \\
\hline Day 3 & $97.9 \pm 145.3$ & $36.5 \pm 51.7$ & $<0.001$ & $19.4 \pm 12.2$ & $16.4 \pm 7.9$ & 0.079 \\
\hline Day 7 & $44.1 \pm 40.0$ & $26.1 \pm 17.0$ & $<0.001$ & $26.0 \pm 24.5$ & $21.7 \pm 18.7$ & 0.214 \\
\hline \multicolumn{7}{|c|}{ Bilirubin (mg/dL)* } \\
\hline Baseline & $0.8 \pm 0.3$ & $0.6 \pm 0.3$ & $<0.001$ & $0.7 \pm 0.3$ & $0.7 \pm 0.3$ & 0.353 \\
\hline Day 1 & $1.1 \pm 0.5$ & $0.9 \pm 0.4$ & $<0.001$ & $0.9 \pm 0.5$ & $0.9 \pm 0.6$ & 0.575 \\
\hline Day 3 & $1.1 \pm 0.8$ & $0.8 \pm 0.3$ & $<0.001$ & $0.8 \pm 0.4$ & $0.7 \pm 0.5$ & 0.126 \\
\hline Day 7 & $0.9 \pm 0.9$ & $0.6 \pm 0.3$ & $<0.001$ & $0.6 \pm 0.2$ & $0.6 \pm 0.6$ & 0.73 \\
\hline \multicolumn{7}{|c|}{$\begin{array}{l}\text { Abnormal elevation of LFTs } \\
(\geq \text { Grade } 2)\end{array}$} \\
\hline $\mathrm{AST}$ & $76(38 \%)$ & $11(9 \%)$ & $<0.001$ & $7(2 \%)$ & $1(2 \%)$ & 0.857 \\
\hline $\mathrm{ALT}$ & $80(40 \%)$ & $9(8 \%)$ & $<0.001$ & $5(2 \%)$ & $1(2 \%)$ & 0.893 \\
\hline Bilirubin & $25(13 \%)$ & $3(3 \%)$ & 0.002 & $11(3 \%)$ & $3(5 \%)$ & 0.472 \\
\hline Total & $99(50 \%)$ & $14(12 \%)$ & $<0.001$ & $19(6 \%)$ & $5(9 \%)$ & 0.384 \\
\hline
\end{tabular}

*Values are means \pm SD. LG: laparoscopic gastrectomy; OG: open gastrectomy; LC: laparoscopic colectomy; OC: open colectomy; p1: LG versus OG; p2. LC versus OC; LFTs: liver function tests; AST: aspartate aminotransferase; ALT: alanine aminotransferase. 
Table 5 Results of univariate and multivariate analyses for postoperative abnormal elevation of LFTs in 319 gastric cancer patients.

\begin{tabular}{|c|c|c|c|c|c|}
\hline \multirow[b]{2}{*}{ Variable } & \multirow[b]{2}{*}{ Category } & \multicolumn{2}{|c|}{ Univariate analysis } & \multicolumn{2}{|c|}{ Multivariate analysis } \\
\hline & & OR $(95 \% \mathrm{CI})$ & $p$ value & OR $(95 \% \mathrm{CI})$ & $p$ value \\
\hline Procedure & LG vs $\mathrm{OG}$ & $7.50(4.14-14.49)$ & $<0.001$ & $7.99(3.69-18.85)$ & $<0.001$ \\
\hline Age & per years & $0.99(0.97-1.01)$ & 0.186 & $0.99(0.97-1.02)$ & 0.449 \\
\hline Gender & Male vs Female & $0.63(0.39-1.01)$ & 0.057 & $0.65(0.37-1.14)$ & 0.133 \\
\hline Body mass index & per $\mathrm{kg} / \mathrm{m}^{2}$ & $1.07(0.99-1.14)$ & 0.050 & $1.08(1.00-1.18)$ & 0.052 \\
\hline ASA-PS & 2 vs 1 & $0.75(0.45-1.24)$ & 0.264 & & \\
\hline Serum albumin & per g/dL & $2.07(1.21-3.67)$ & 0.007 & $1.51(0.76-3.08)$ & 0.242 \\
\hline Alcohol use & $(+)$ vs $(-)$ & $1.28(0.81-2.03)$ & 0.298 & & \\
\hline Cardiovascular disease & $(+)$ vs $(-)$ & $0.67(0.30-1.41)$ & 0.300 & & \\
\hline Diabetes mellitus & $(+)$ vs $(-)$ & $1.23(0.65-2.30)$ & 0.516 & & \\
\hline $\begin{array}{l}\text { Neoadjuvant } \\
\text { chemotherapy }\end{array}$ & $(+) \mathrm{vs}(-)$ & $0.93(0.47-1.80)$ & 0.842 & & \\
\hline Extent of resection & Total vs Distal & $1.03(0.64-1.64)$ & 0.918 & & \\
\hline Cholecystectomy & $(+) \mathrm{vs}(-)$ & $0.23(0.10-0.48)$ & $<0.001$ & $0.48(0.18-1.20)$ & 0.128 \\
\hline $\mathrm{T}$ & $\mathrm{T} 3,4$ vs T0-2 & $0.71(0.43-1.14)$ & 0.158 & $2.14(0.99-4.76)$ & 0.056 \\
\hline $\mathrm{N}$ & N1-3 vs N0 & $0.71(0.44-1.13)$ & 0.148 & $1.23(0.61-2.49)$ & 0.570 \\
\hline Operation time & per minute & $1.00(1.00-1.01)$ & 0.038 & $1.00(0.99-1.00)$ & 0.192 \\
\hline
\end{tabular}

LFTs: liver function tests; OR: odds ratio; CI: confidence interval; LG: laparoscopic gastrectomy; OG: open gastrectomy, ASA:

American Society of Anesthesiology Classification; B-I: Billroth-I; R-Y: Roux-en-Y. 